\title{
Determinación de las tasas de oxidación, nitrificación y sedimentación en el proceso de autopurificación de un río de montaña
}

\author{
Determination of oxidation rates, nitrification and sedimentation \\ in the process of self-purification of a mountain river \\ Jorge Virgilio Rivera Gutiérrez ${ }^{1}$ \\ Recibido 29 de octubre de 2014, aceptado 14 de septiembre de 2015 \\ Received: October 29, 2014 Accepted: September 14, 2015
}

\begin{abstract}
RESUMEN
Este artículo presenta la evaluación de la oxidación de la materia orgánica presente en el agua mediante la determinación de tasas cinéticas que indican la velocidad a la cual el río pierde o gana oxígeno y también las tasas de nitrificación y decaimiento de patógenos. El cálculo de las tasas define el proceso de autopurificación o capacidad de asimilación de la carga orgánica en el río Frío, fuente principal de potabilización y riego de los municipios de Floridablanca y Girón en el Departamento de Santander, Colombia. Se modelaron $26,6 \mathrm{~km}$ de río, es decir, la totalidad del canal principal de la microcuenca, cuya característica es ser un río de montaña muy oxigenado en su zona alta, pero contaminado en la zona baja, en la que se captan 26,2 ton $\mathrm{d}^{-1}$ de material biodegradable proveniente de vertidos domésticos. El cálculo de dichas tasas se desarrolla por medio de correlaciones y análisis numérico de las concentraciones en cada uno de los (7) tramos del río. Se desarrollaron estudios de coeficiente de dispersión, análisis morfométricos e hidráulicos, y a su vez se modeló el comportamiento de oxígeno, carbono, nitrógeno, patógenos y sólidos suspendidos con el QUAL2K, con el fin de corroborar el comportamiento de las tasas calculadas. Conocer la autopurificación de las fuentes hídricas es fundamental para establecer estrategias de sostenibilidad.
\end{abstract}

Palabras clave: Autopurificación, tasas de oxidación, nitrificación, sedimentación, QUAL2K, río de montaña.

\begin{abstract}
This paper presents the evaluation of oxidation of organic matter in water via the determination of kinetic rate indicating the frequency at which the stream loses or gains oxygen and nitrification rates and the decay of pathogens. The fee calculation defines the process of auto purification and assimilative capacity of the organic load on the Frio River, the main source of potable and irrigation of the municipalities of Floridablanca and Girón in the Department of Santander, Colombia. $26.6 \mathrm{~km}$ of river were modeled, $i e$, the entire main channel basin, which characteristic is to be a highly oxygenated mountain river in its upper part, but in the low polluted area, which capture 26.2 Ton. $d^{-1}$ of biodegradable material from household waste. The calculation of these rates is developed through correlations and numerical analysis of concentrations in each of the (7) reach of the river. The model of behavior of: Oxigen, carbon, nitrogen, pathogens and suspended solids through QUAL2K, in order to corroborate the behavior of the calculated rates: studies of dispersion coefficient, morphometric and hydraulic analyzes were developed. Knowing the auto purification of water sources is critical to establishing sustainability strategies.
\end{abstract}

Keywords: Self-purification, oxidation rates, nitrification, sedimentation, QUAL2K, mountain rive.

1 Dirección de Investigaciones. Unidades Tecnológicas de Santander. 6917700 Ext. 2007. Bucaramanga, Colombia. E-mail: jrivera@correo.uts.edu.co 


\section{INTRODUCCIÓN}

Los cuerpos hídricos del Área Metropolitana de Bucaramanga: Suratá, Tona, Oro, Frío y Lato, ubicados en las cabeceras de los municipios Lebrija, Girón, Bucaramanga, Floridablanca y Piedecuesta, respectivamente, presentan contaminación por materia orgánica y metales pesados, convirtiéndose en una emergencia ambiental. La falta de cultura ambiental, el desarrollo industrial y el aumento poblacional del área metropolitana (1.089.269 habitantes a 2015), según [1] permiten un aumento progresivo de contaminantes a estos ríos, los que presentan dos objetivos: fuente de captación y cuerpo receptor. Según la red de monitoreo de la Corporación Autónoma Regional Para la Defensa de la Meseta de Bucaramanga (CDMB) [2], menciona que una de las afluentes más importantes de la subcuenca Oro, es el río Frío, evaluada en (4) estaciones: Esperanza, Pórtico, Totumos y Caneyes, correspondiente a RF-03, RF-P, RF-B, RF-1A, respectivamente, en forma descendente. Aclaran que el río Frío es una de las fuentes más contaminadas del área metropolitana, debido al efluente tratado de la única Planta de aguas residuales del área, denominada "PTAR Río Frío" de Floridablanca y los vertidos crudos. Los demás municipios vierten sus aguas directo al río de Oro. La mitigación de la contaminación orgánica es urgente y requiere establecer la carga asimilable por los ríos afectados, en este caso el río Frío. El río presenta tres zonas: la zona alta, de calidad óptima para consumo humano (OD = $\left.8 \mathrm{mg} . \mathrm{L}^{-1}, \mathrm{DBO}=2 \mathrm{mg} . \mathrm{L}^{-1}\right)$, donde se captan 0,5 $\mathrm{m}^{3} \cdot \mathrm{s}^{-1}$, para el acueducto de Floridablanca, dejando $1,4 \mathrm{~m}^{3} \cdot \mathrm{s}^{-1}$. La zona media, el caudal se aumentan a $1,8 \mathrm{~m}^{3} \cdot \mathrm{s}^{-1}$ debido a la confluencia de la quebrada Mensuly, ubicada en el asentamiento urbano de Floridablanca. La zona baja, se encuentran la mayor carga orgánica generada por el efluente de la PTAR y los vertidos crudos (By-pass del alcantarillado Floridablanca y Angelina), además se presenta una fuente de dilución, denominada Quebrada Aranzoque, sumando en el canal un caudal de $2,5 \mathrm{~m}^{3} \cdot \mathrm{s}^{-1}$, con el que confluye al río de Oro en la zona de Caneyes en el municipio de Girón. Los vertidos que confluyen de manera continua contienen concentraciones de $80 \mathrm{mg} . \mathrm{L}^{-1}$ y $400 \mathrm{mg} . \mathrm{L}^{-1}$ de DBO entre tratados y crudos, respectivamente. La PTAR río Frío solo trata el 5\% de las aguas residuales de Bucaramanga y el $80 \%$ de las de Floridablanca. Según [3], en un estudio realizado mediante la modelación de la calidad del agua en la zona baja del río Frío. El río Frío genera una carga de 61,9 ton $\mathrm{d}^{-1}$, solo diluye 16,85 ton $\mathrm{d}^{-1}$, lo que permite una acumulación de materia orgánica en la zona baja, convirtiendo el río en una cloaca que afecta constantemente el río de Oro. Controlar la capacidad de autopurificación es una de las alternativas más viables para alcanzar la sostenibilidad de dicha fuente hídrica.

El estudio se enfoca en evaluar cuánta carga orgánica puede asimilar el río Frío de acuerdo con las condiciones morfométricas y la cinética de oxidación de dicha carga orgánica. La carga orgánica o materia orgánica está definida por carbono, nitrógeno, patógenos y sólidos suspendidos. Las tasas cinéticas que se estudiaron son: tasa de desoxigenación, tasa de reaireación, tasa de nitrificación, tasa de sedimentación, la tasa de decaimiento de patógenos y tasa de remoción de materia particulada. Según, [4], para evaluar la autopurificación en ríos de montaña, deben tenerse en cuenta estas tasas con el fin de aplicarlas a modelos hidráulicos y de calidad con el fin de simular la capacidad de asimilación de determinantes tales como la DBO, los patógenos y los sólidos suspendidos en el río.

En la Figura 1 se ilustra la zona de estudio, ubicada entre los municipios de Floridablanca y Girón en los meridianos $73^{\circ} 2^{\prime}$ y $73^{\circ} 9^{\prime}$ de longitud y los paralelos $7^{\circ} 7^{\prime}$ y $7^{\circ} 3^{\prime}$ de latitud norte en el departamento de Santander, Colombia.

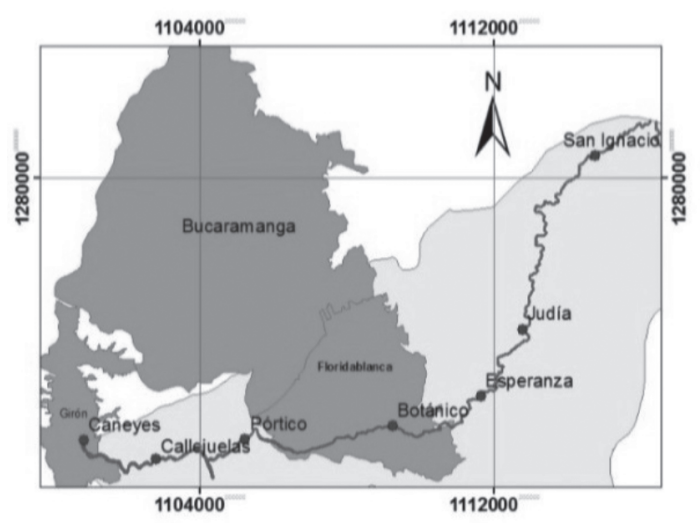

Figura 1. Ubicación del río Frío, Santander, Col.

Las variables de calidad son simuladas en un perfil longitudinal del río que contiene una cabecera, como punto de partida de dicha simulación y un downstream o fin del segmento hidráulico simulado. 
El perfil longitudinal, como se ilustra en la Figura 2 , presenta una pendiente de $7 \%$ en la zona alta y $1 \%$ en la zona baja del segmento, con una caída de altura que oscila entre 2800 a $400 \mathrm{msnm}$, permitiendo una disminución de la velocidad y aumento de la temperatura $\left(16\right.$ a $\left.31^{\circ} \mathrm{C}\right)$.

El segmento hidráulico se discretiza en (7) tramos denominados como: San Ignacio, Judía, Esperanza, Jardín Botánico, Pórtico, Callejuelas y Caneyes, en un trayecto $26,6 \mathrm{~km}$ y un tiempo de viaje de 11,7 horas.

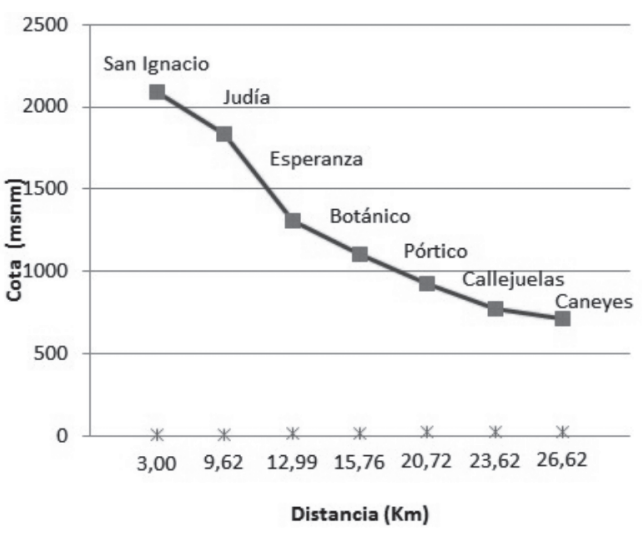

Figura 2. Perfil longitudinal de altura- R Frío.

\section{ANÁLISIS TEÓRICO}

La determinación de la asimilación de la carga contaminante en un río de montaña obedece según [4] a las siguientes actividades: 1) Investigación preliminar y levantamiento de información. 2) Caracterización hidráulica mediante ensayos con trazadores. 3). Estimación preliminar de la tasa de reaireación. 4) Estimación preliminar de las tasas de degradación de materia orgánica y nitrificación. 5) Estimación preliminar de la tasa de mortalidad de patógenos y la velocidad de sedimentación de sólidos suspendidos y materia orgánica particulada. 6) Implementación y calibración de un modelo de calidad de agua predictivo. 7) Evaluación de la capacidad de autopurificación y asimilación de la carga contaminante. Se tomaron muestras puntuales en la mitad de cada tramo, de acuerdo con tiempo de viaje del río y un periodo de retorno de 10 años, se cumplió con las cadenas de custodia y protocolos de monitoreo de acuerdo con las normas OMM e ICONTEC, [5, 6]. Las muestra fueron filtradas para determinar DBO y DQO diluidas y la materia orgánica particulada (MOP=detritos). La toma de muestras se realizó en época de estiaje (junio 2012) y los análisis fisicoquímicos y microbiológicos fueron realizados en el laboratorio acreditado de la CDMB.

\section{Caracterización hidráulica}

Se realizó un estudio hidráulico con la información morfométrica obtenida en cada uno de los tramos, como se ilustra en la Tabla 1. Los aforos se compararon con las curvas de gastos para 10 años de retorno. Fue necesaria la información de las estaciones hidrométricas de la CDMB tales como Esperanza "RF-03"; Pórtico "RF-P" y Caneyes "RF-1A", también se crearon nuevas estaciones en la zona alta y baja denominadas San Ignacio, Judía, Botánico y Callejuelas, fueron georrefenciadas y validadas sus características hidráulicas como: velocidad media y caudal, al igual que las características geométricas como ancho, profundidad, área transversal y pendiente. En la Figura 3 se ilustra la discretización de los 7 tramos en el río Frío.

Tabla 1. Morfometría del segmento hidráulico río Frío.

\begin{tabular}{|c|c|c|c|c|c|}
\hline \multicolumn{2}{|c|}{ COORDENADAS } & $\mathbf{X}$ & $\mathbf{U}$ & $\mathbf{H}$ & $\mathbf{Q}$ \\
\hline Lat $(\mathbf{N})$ & Lon $(\mathbf{E})$ & $\mathbf{K m}$ & $\mathbf{m} / \mathbf{s}$ & $\mathbf{m}$ & $\mathbf{m}^{3} / \mathbf{s}$ \\
\hline 1280580 & 1114780 & 3,0 & 0,6 & 0,2 & 0,9 \\
\hline 1276021 & 1112782 & 6,6 & 0,5 & 0,5 & 0,9 \\
\hline 1274252 & 1111654 & 3,4 & 0,6 & 0,3 & 1,9 \\
\hline 1273476 & 1109245 & 2,8 & 0,6 & 0,2 & 1,4 \\
\hline 1273120 & 1105215 & 5,0 & 0,6 & 0,3 & 1,8 \\
\hline 1272617 & 1102788 & 2,9 & 0,9 & 0,3 & 2,4 \\
\hline 1273097 & 1100822 & 3,0 & 0,8 & 0,3 & 2,5 \\
\hline
\end{tabular}

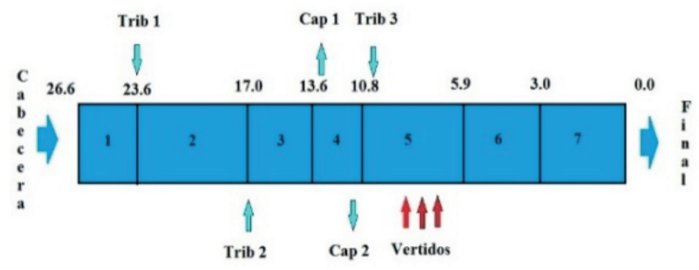

Figura 3. Modelo Conceptual hidráulico del río.

\section{Estimación de la tasa de desoxigenación}

La dinámica del oxígeno en los cuerpos lóticos se evidencia por la ganancia o pérdida de la concentración del gas diluido en la columna de agua. El río presenta tres fases (aire/agua/fondo), en 
la cuales se presentan flujos de oxígeno generadas por el consumo de este en procesos de oxidación de materia orgánica presente en la columna y el fondo, denominado bento, también es demandado por contaminantes no biodegradables oxidables presentes en desechos industriales.

En el presente estudio se investiga la tasa a la que se pierde el oxígeno por acción de la carga orgánica. La tasa es calculada aplicando tres técnicas: 1) la botella winkler, 2) aplicando la ecuación de Hydroscience y 3) la aplicación del carbono orgánico total. Respecto de la técnica de la botella, se evalúa el consumo de oxígeno, determinando el cambio en la concentración de la DBO (y), durante 21 días de incubación a $20^{\circ} \mathrm{C}$, la ecuación que rige el fenómeno es la siguiente:

$$
\frac{d L_{t}}{d t}=-K^{*} L_{t}
$$

Donde $L_{t}=\mathrm{DBO}$ remanente en el agua para un tiempo $t$, expresada en $\left(\mathrm{mgO}_{2} \mathrm{~L}^{-1}\right) ; K=$ Tasa de desoxigenación o tasa de oxidación de la DBO expresada en $\left(\mathrm{d}^{-1}\right)$ y $t=$ tiempo de oxidación en (días).

El cálculo de la tasa se desarrolla de acuerdo con la aplicación de mínimos cuadrados sobre los datos obtenidos de $\mathrm{DBO}$ en el período de incubación. De acuerdo con la ecuación: $y=L\left(1-e^{-k t}\right)$; en forma diferencial sería $\frac{d y}{d t}=K(L-y)$; donde $L=$ es la

DBO remanente en el agua para el tiempo $t=0$, o también llamada demanda bioquímica de oxígeno carbonácea última, DBOCu, $y=\mathrm{DBO}$ ejercida. Para la aplicación de los mínimos cuadrados se usan las ecuaciones:

$$
\begin{gathered}
N^{*} a+b \sum y-\sum y^{\prime}=0 \\
a \sum y+b \sum y^{2}-\sum y y^{\prime}=0
\end{gathered}
$$

Donde $N$ es el número de pares de valores observados -1. Como el delta de tiempo entre las mediciones no fue constante, se da lugar a la utilización de dos tipos de ecuación para calcular la $y^{\prime}$.

Para con $\Delta t$ constante, se usa:

$$
\frac{d y}{d t}=y^{\prime}=\frac{y_{N+1}-y_{N-1}}{2 \Delta t}
$$

Para $\Delta t$, variable, se usa:

$$
y^{\prime}=\frac{\left(y_{N}-y_{N-1}\right)\left(\frac{t_{N+1}-t_{N}}{t_{N}-t_{N-1}}\right)+\left(y_{N+1}-y_{N}\right)\left(\frac{t_{N}-t_{N-1}}{t_{N+1}-t_{N}}\right)}{t_{N+1}-t_{N-1}}
$$

Donde: $y_{N}$ y $T_{N}=$ son la magnitud y tiempo del punto para el que se calcula $y^{\prime} ; y_{N-1}$ y $t_{N-1}=$ Valores del punto anterior; $y_{N+1}$ y $t_{N+1}=$ Valores del punto posterior. Finalmente se obtiene la tasa aplicando, Obteniéndose un valor en base neperiana en unidades de $\left(d^{-1}\right)$.

$$
-b=-K=\frac{n^{*} \sum y y^{\prime}-\sum y^{\prime *} \sum y}{\left(\sum y\right)^{2}-N^{*} \sum y^{2}}
$$

En la Tabla 2 se describe el comportamiento de la tasa para los 7 tramos, aplicando este método.

Tabla 2. Correlación de K1 (botella).

\begin{tabular}{|c|c|c|}
\hline TRAMO & $\left.\mathbf{K d ~} \mathbf{d d}^{\mathbf{- 1}}\right)$ & $\mathbf{R 2}$ \\
\hline Ignacio & 0,03 & 0,60 \\
\hline Judía & 0,01 & 0,42 \\
\hline Esperanza & 0,04 & 0,53 \\
\hline Botánico & 0,04 & 0,73 \\
\hline Pórtico & 0,01 & 0,60 \\
\hline Callejuelas & 0,03 & 0,37 \\
\hline Caneyes & 0,02 & 0,43 \\
\hline
\end{tabular}

La segunda forma de calcular la tasa de desoxigenación es aplicando las ecuaciones de [7] y Bosko para profundidades $0 \leq H \leq 8$ pies, la tasa sería: $k_{d}=0,3\left[\frac{H}{8}\right]^{-0,434}$, mientras que para $H>8$ pies; la tasa $k_{d}=0,3$. Es de aclarar que en nuestro caso el río Frío, presentó una profundidad máxima de 0,5 m, es decir (1,64 pies). La tasa puede ser extrapolada al usar otra temperatura, así: $k=k_{20} \theta^{T-20}$ Donde: $k$, es la Tasa a temperatura (T), $k_{20}=$ Tasa de descomposición a $20^{\circ} \mathrm{C} ; \theta=$ Constante $=1,047$. La DBO removida tiende a incrementarse con la temperatura y a incrementarse aguas abajo de fuentes puntuales. La sedimentación mejora con aguas poco profundas, en sistemas generalmente de baja profundidad existe una mayor tasa de remoción de DBO, como se ilustra en la Tabla 3.

La tercera técnica que se utilizó es la aplicación del carbono orgánico total COT en cada tramo del río para convertirlo a DBOC última, partiendo del factor 
Tabla 3. Validación de la Kd (desoxigenación).

\begin{tabular}{|c|c|c|c|c|}
\hline \multirow{2}{*}{ TRAMO } & Hydro & Bosko & Botella & Exp \\
\cline { 2 - 5 } & Kd d $^{-\mathbf{1}}$ & Kd d $^{\mathbf{1}}$ & Kd d $^{\mathbf{1}}$ & Kd d $^{\mathbf{1}}$ \\
\hline Ignacio & 2,32 & 0,01 & 0,03 & 0,50 \\
\hline Judía & 1,70 & 0,01 & 0,01 & 0,59 \\
\hline Esperanza & 2,03 & 0,01 & 0,04 & 0,51 \\
\hline Botánico & 2,49 & 0,01 & 0,04 & 0,43 \\
\hline Pórtico & 2,26 & 0,14 & 0,01 & 0,15 \\
\hline Callejuelas & 2,09 & 0,07 & 0,03 & 0,18 \\
\hline Caneyes & 2,09 & 0,01 & 0,02 & 0,27 \\
\hline
\end{tabular}

estequiométrico, 2,67 $\mathrm{gO}_{2} / 1 \mathrm{gC}$, establecido para un sustrato de amonio. En la Tabla 4 se nota cómo cambia dicha tasa respecto del COT presente en el agua. La tasa $k_{d} ;\left(\mathrm{d}^{-1}\right)$ se calcula logarítmicamente por la relación de la $\mathrm{DBOC}_{5}$, con dicha $\mathrm{DBOCu}$, de acuerdo con la ecuación (7):

$$
-k_{d}=\operatorname{Ln}\left[\frac{D_{B O C}}{D B O C U}\right] * \frac{1}{5}
$$

Tabla 4. Cálculo de la Kd experimental.

\begin{tabular}{|c|c|c|c|c|}
\hline \multirow{2}{*}{ ID } & DBO5 & COT & DBOCU & Kd Exp \\
\cline { 2 - 5 } & $\mathbf{m g} / \mathbf{L}$ & $\mathbf{m g} / \mathbf{L}$ & $\mathbf{m g} / \mathbf{L}$ & $\mathbf{d}^{\mathbf{- 1}}$ \\
\hline 1 & 1,3 & 5,9 & 15 & 0,50 \\
\hline 2 & 1,4 & 9,9 & 26 & 0,59 \\
\hline 3 & 1,5 & 7,1 & 19 & 0,51 \\
\hline 4 & 1,8 & 5,9 & 16 & 0,43 \\
\hline 5 & 8,4 & 6,6 & 18 & 0,15 \\
\hline 6 & 58,0 & 54,5 & 146 & 0,18 \\
\hline 7 & 49,0 & 69,9 & 186 & 0,27 \\
\hline
\end{tabular}

\section{Estimación de la tasa de reaireación}

Se conoce como reaireación el proceso por el que el oxígeno y demás componentes gaseosos del aire son renovados en la columna de agua, debido al movimiento del río. Los patrones que la controlan son diferentes de los que afectan la desoxigenación. Es importante, en consecuencia, estudiar estos patrones, así como los procedimientos para predecir las tasas reales de reaireación. Si por alguna razón el nivel de OD en el agua es menor que el valor de saturación, el agua disuelve más oxígeno de la atmósfera y se acerca nuevamente al nivel de saturación. Según la ley de Henry, a temperatura constante, la solubilidad de un gas en un líquido es proporcional a la presión parcial del gas. La tasa de solubilización del oxígeno es proporcional al déficit de saturación, como lo indica la ecuación: $\frac{d D}{d t}=-K_{a} D$
Integrando $D=D o * e^{-K_{a} T}$. Donde: $D=$ déficit de OD para el tiempo t, mg/L; $D o=$ déficit inicial de OD, $\mathrm{mg} / \mathrm{L} ; K_{\alpha}=$ constante de reaireación, base natural, $\mathrm{d}^{-1}$. Expresando la ecuación en términos de concentraciones de OD, se obtiene:

$$
\operatorname{Ln}\left(\frac{(C s-C)}{(C s-C O)}\right)=-K_{a} t
$$

Donde: $C=$ concentración de OD para el tiempo t, mg/L; $C O=$ concentración inicial $\mathrm{OD}, \mathrm{mg} / \mathrm{L}$; $C s=$ concentración de saturación de $\mathrm{OD}, \mathrm{mg} / \mathrm{L}$. La validación de la tasa de reaireación aplicando la metodología de saturación de oxígeno, se ilustra en la Tabla 5.

Tabla 5. Tasa de reaireación experimental.

\begin{tabular}{|c|c|c|c|c|}
\hline \multirow{2}{*}{ TRAMO } & $\mathbf{C t}$ & $\mathbf{C o}$ & $\mathbf{C s}$ & $\mathbf{K a}$ \\
\cline { 2 - 5 } & $\mathbf{m g L}^{\mathbf{- 1}}$ & $\mathbf{m g L}^{\mathbf{- 1}}$ & $\mathbf{m g L}^{\mathbf{- 1}}$ & $\mathbf{d}^{\mathbf{- 1}}$ \\
\hline Ignacio & 7,80 & 8,10 & 8,16 & 6,4 \\
\hline Judía & 7,10 & 7,20 & 7,31 & 1,6 \\
\hline Esperanza & 7,00 & 7,05 & 7,34 & 1,0 \\
\hline Botánico & 6,90 & 6,95 & 7,30 & 1,1 \\
\hline Pórtico & 6,80 & 6,85 & 7,02 & 0,8 \\
\hline Callejuelas & 3,16 & 4,98 & 6,50 & 1,7 \\
\hline Caneyes & 1,80 & 2,48 & 6,55 & 3,3 \\
\hline
\end{tabular}

La solubilización del oxígeno puede ocurrir solo en la interfaz aire-agua, donde una película delgada de agua es rápidamente saturada. La tasa de reaireación posterior depende de la difusión del oxígeno mediante el cuerpo de agua, la que es muy lenta. En ríos turbulentos la capa superficial saturada se rompe continuamente y el proceso de reaireación se efectúa más rápido. La otra forma de calcular la tasa de reaireación es usando ecuaciones estocásticas de manera preliminar. Se han desarrollado varias ecuaciones basadas en estudios de ríos, con muy diferentes características hidráulicas. Entre las más comunes se citan las siguientes:

$$
\begin{aligned}
K_{a} & =3,93 * \frac{U^{0.5}}{H^{1,5}} \\
K_{a} & =5,03 * \frac{U^{0,969}}{H^{1,673}} \\
K_{a} & =5,32 * \frac{U^{0,67}}{H^{1,85}}
\end{aligned}
$$




$$
\begin{gathered}
K_{a}=C^{*} S^{*} U \\
S=\frac{\left.\left(U^{*}\right)\right)}{H^{4 / 3}} \\
K_{a}=\frac{1,923 * U^{0,273}}{H^{0,894}}
\end{gathered}
$$

Donde: $U=$ velocidad media $\left(\mathrm{m}, \mathrm{s}^{-1}\right) ; H=$ profundidad media $(\mathrm{m}) ; S=$ pendiente de la línea de energía $\left(\mathrm{m} . \mathrm{m}^{-1}\right) ; C=$ coeficiente de escape $\left(\mathrm{m}^{-1}\right)$ se determina experimentalmente, se recomienda: $C=0,177 \mathrm{~m}^{-1}$, a $20^{\circ} \mathrm{C}$ para $0,42<\mathrm{Q}<85 \mathrm{~m}^{3} . \mathrm{s}^{-1}$; o $C=0,361 \mathrm{~m}^{-1}$, a $20^{\circ} \mathrm{C}$ para $0,42<\mathrm{Q}<85 \mathrm{~m}^{3} \cdot \mathrm{s}^{-1} ; n=$ coeficiente Manning, $\left(\mathrm{m}^{-1 / 3} \cdot \mathrm{s}\right)$.

En la Tabla 6 se compara reaireación de los 7 tramos de acuerdo con cada una de las ecuaciones empíricas anteriormente mencionadas.

Tabla 6. Cálculo de la Ka por ecuaciones.

\begin{tabular}{|c|c|c|c|c|c|c|}
\hline \multirow{2}{*}{ TRAMO } & $\mathbf{E x p}$ & $\mathbf{( 1 )}$ & $\mathbf{( 2 )}$ & $\mathbf{( 3 )}$ & $\mathbf{( 4 )}$ & $\mathbf{( 5 )}^{*}$ \\
\cline { 2 - 7 } & $\mathbf{d}^{\mathbf{- 1}}$ & $\mathbf{d}^{\mathbf{- 1}}$ & $\mathbf{d}^{\mathbf{- 1}}$ & $\mathbf{d}^{\mathbf{- 1}}$ & $\mathbf{d}^{\mathbf{1}}$ & $\mathbf{d}^{\mathbf{- 1}}$ \\
\hline Ignacio & 6,4 & 5,9 & 0,1 & 31,6 & 51,7 & 2,3 \\
\hline Judía & 1,6 & 3,1 & 0,0 & 9,5 & 13,7 & 0,2 \\
\hline Esperanza & 1,0 & 4,6 & 0,1 & 20,6 & 31,2 & 2,0 \\
\hline Botánico & 1,1 & 7,1 & 0,1 & 47,5 & 76,9 & 4,2 \\
\hline Pórtico & 0,8 & 5,9 & 0,1 & 33,2 & 51,5 & 0,5 \\
\hline Callejuelas & 1,7 & 5,5 & 0,1 & 34,4 & 46,5 & 0,8 \\
\hline Caneyes & 3,3 & 5,4 & 0,1 & 31,5 & 43,7 & 0,3 \\
\hline
\end{tabular}

*(1) Texas Water Development Board, 1970 [12]; (2) O'Connor, 1958 [8]; (3) Churchill, M. A., Elmore, H. L y Buckingham, R.A, 1962 [9]; (4) Owens,.M., Edwards, R., y Gibbs, 1964 [10]; (5) Tsivoglou, E. C. y Wallace, S. R, 1972 [11].

\section{Estimación de la tasa de nitrificación}

La demanda bioquímica de oxígeno nitrogenada, DBON, se genera por la oxidación del nitrógeno Kjeldahl, NTK, (N orgánico + amonio), en el proceso denominado "nitrificación", de acuerdo con las reacciones de Gaudy [13]. Primero, las bacterias Nitrosomonas convierten el ion amonio a nitrito.

$$
\mathrm{NH}_{4}^{+}+1,5 \mathrm{O}_{2} \rightarrow 2 \mathrm{H}^{+}+\mathrm{H}_{2} \mathrm{O}+\mathrm{NO}_{2}^{-}
$$

Segundo, la bacteria Nitrobacter convierte el nitrito en nitrato.

$$
\mathrm{NO}_{2}^{-}+0,5 \mathrm{O}_{2} \rightarrow \mathrm{NO}_{3}^{-}
$$

De esta manera el oxígeno consumido para los dos estados se puede calcular.

$$
\begin{aligned}
& r_{o a}=\frac{1,5(32)}{14}=3,43 \mathrm{gO} \mathrm{gN}^{-1} \\
& r_{o i}=\frac{0,5(32)}{14}=1,14 \mathrm{gO} \mathrm{gN}^{-1}
\end{aligned}
$$

Donde: $r_{o a}$ oxígeno consumido para la conversión de amonio a nitrito; $r_{o i}$ oxígeno consumido para la conversión de nitrito a nitrato. Todo el proceso se puede representar como: $r_{o n}=r_{o a}+r_{o i}=4,57 \mathrm{gOgN} \mathrm{g}^{-1}$.

Donde $r_{o n}=$ es la cantidad de oxígeno consumido por unidad de nitrógeno oxidado en todo el proceso de nitrificación. Para que exista la nitrificación deben existir los siguientes factores: 1) presencia suficiente de bacterias nitrificantes. 2) un $\mathrm{pH}$ alcalino, alrededor de 8 y 3 ) suficiente oxígeno ( 1 a $\left.2 \mathrm{mg} \mathrm{L}^{-1}\right)$.

Las ecuaciones se validan con la información recogida en campo en tiempo real (tiempo de viaje del río Frío) en cada tramo. $\mathrm{La} \mathrm{DBON}=L_{N}$, se calcula aplicando el factor estequiométrico: $L_{N}=4,57$ NTK. Para un sistema de flujo inyectado, el balance de masa para la DBON y el déficit de oxígeno se puede calcular suponiendo que $L_{N}=L_{N o}$ y $D=D_{o}$ para un tiempo $t=0$, así:

$$
L_{N}=L_{N o} * e^{-\frac{K_{n}}{U} x}
$$

El déficit de oxígeno se podría calcular como:

$$
D=D_{o} * e^{-\frac{K_{a}}{U} x}+\frac{K_{n} L_{o}}{K_{a}-K_{n}}\left(e^{-\frac{K_{n}}{U} x}-e^{-\frac{K_{a}}{U} x}\right)
$$

Donde: $D=$ Déficit de oxígeno, $\left(\mathrm{mgO}_{2} \cdot \mathrm{L}^{-1}\right) ; D_{o}=$ Déficit de oxígeno inicial $\left(\mathrm{mgO}_{2} \cdot \mathrm{L}^{-1}\right) ; K_{n}=$ constante de nitrificación, base natural, $\mathrm{d}^{-1}$. La tasa se calcula integrando por separado las concentraciones de nitrógeno en cada tramo para un tiempo $T$, de retención, mediante la ecuación (18):

$$
\operatorname{Ln}\left(\frac{L_{N}}{L_{N o}}\right) \frac{U}{X}=-K_{n}
$$

De acuerdo con la Tabla 7 , el valor de $K_{n}$ generalmente se presenta un rango entre $0,1 \mathrm{y} 0,5 \mathrm{~d}^{-1}$, ahora bien, para ríos profundos los valores de $K_{n}$ pueden llegar a tener valores cercanos a $1,5 \mathrm{~d}^{-1}$, según Camacho [4] la tasa de nitrificación en ríos de montaña para Colombia es muy cercana a $1,2 \mathrm{~d}^{-1}$, de acuerdo con un estudio de comparación de métodos de validación. 
Tabla 7. Tasa de Nitrificación.

\begin{tabular}{|c|c|c|}
\hline \multirow{2}{*}{ TRAMO } & NTK & Kn \\
\cline { 2 - 3 } & $\mathbf{m g ~ N / L}$ & $\mathbf{d}^{\mathbf{1}}$ \\
\hline Ignacio & 0,74 & 0,88 \\
\hline Judía & 0,75 & 0,09 \\
\hline Esperanza & 0,78 & 0,60 \\
\hline Botánico & 0,81 & 0,74 \\
\hline Pórtico & 2,72 & 13,5 \\
\hline Callejuelas & 28,40 & 63,6 \\
\hline Caneyes & 30,50 & 1,71 \\
\hline
\end{tabular}

\section{Estimación de la tasa de decaimiento de patógenos}

Muchos de los microrganismos se encuentran dentro del cuerpo y se accede a otros por la piel o por ingesta de agua sin tratar. Los tipos de bacterias que pueden representar la mayoría de estos microorganismos son: 1) Coliformes total, CT, larga lista de anaeróbicos, tales como la Escherichia coli, o E-coli, la Aerobacter aerogenes, entre otros. 2) Coliformes fecales, $\mathrm{CF}$, pertenece a los $\mathrm{CT}$, se encuentran en los intestinos de los animales de sangre caliente, sin incluir los terrestres; pueden llegar a ser el $20 \%$ de los CT. 3) Estreptococo fecal, están incluidos en los estreptococos presentes en humanos y animales domésticos. Es muy importante determinar la cantidad de CF, para establecer contaminación fecal y no fecal del total. Las aguas naturales pueden contener entre 100.000 y 400.000 número. $100 \mathrm{~mL}^{-1}$, de fecales.

La tasa de decaimiento de patógenos, según Chapra [14] , se calcula sumando la tasa de mortalidad $K_{b}$; la tasa de pérdida por radiación solar y la tasa de sedimentación de la fracción de patógenos adheribles a las partículas. La salinidad (sólidos y sales disueltas) y la temperatura son otros factores que afectan la mortalidad de patógenos. La tasa de decaimiento de patógenos se puede de representar como:

$$
K_{b}^{\prime}=K_{b 1}+K_{b i}+K_{b s}
$$

Donde: $K_{b}^{\prime}$ tasa de decaimiento total $\left(\mathrm{d}^{-1}\right) ; K_{b 1}$ tasa de mortalidad base $\left(\mathrm{d}^{-1}\right) ; K_{b i}$ tasa de pérdida por radiación solar $\left(\mathrm{d}^{-1}\right) ; K_{b s}$ tasa de pérdida por sedimentación $\left(\mathrm{d}^{-1}\right)$.

Según Thoman [15], la tasa de mortalidad de patógenos se puede calcular como:

$$
K_{b 1}=(0,8+0,02 s) 1,07^{T-20}
$$

Donde $s=$ salinidad (ppt o g.L $\mathrm{L}^{-1}$ ); para agua dulce $s=0$; para aguas de mar, $30<s<35$ ppt. Normalmente la tasa de pérdida se encuentra en un rango de $0,8 \mathrm{~d}^{-1}$, para agua dulce y de $1,4 \mathrm{~d}^{-1}$ para agua de mar.

La tasa de pérdida de patógenos por efectos de la radiación representada por:

$$
K_{b i}=\alpha * \bar{I}
$$

Donde: $\alpha=$ constante de proporcionalidad; $\bar{I}$ energía lumínica $\left(l y \cdot h^{-1}\right)$, de acuerdo a Thomann, $\alpha \approx 1$. En cuanto a la extinción de la luz, se puede modelar de acuerdo a la ley de Beer- Lambert en la cual afirma que la extinción de la luz en el agua es proporcional a la energía lumínica superficial, en función del coeficiente de extinción y la profundidad de acuerdo con la ecuación (22):

$$
I_{(z)}=I_{o} * e^{-K_{e} z}
$$

Donde: $I_{(z)}$ es la energía lumínica $\left(l y \cdot h^{-1}\right) ; l_{o}$ energía lumínica superficial $\left(\mathrm{ly} \cdot \mathrm{h}^{-1}\right) ; K_{e}$ el coeficiente de extinción $\left(\mathrm{m}^{-1}\right) ; z$ profundidad $(\mathrm{m})$. El coeficiente de extinción está en función de la materia particulada y el color en el agua. Si se aplica el disco Secchi (DS) y la profundidad se tiene la siguiente ecuación: $K_{e}=\frac{1,8}{D S}$. De acuerdo a Di Toro [16], si se relacionan los sólidos suspendidos $\left(\mathrm{mg} . \mathrm{L}^{-1}\right)$, quedaría $K e=0,55(S S T)$. La luz promedio $\bar{I}$, se puede calcular integrando con la profundidad del río, así:

$$
\bar{I}=\frac{\int_{0}^{H} I_{0} e^{-K_{e}^{*} z} d z}{H}=\frac{I_{o}}{K_{e} H}\left(1-e^{-K_{e} H}\right)
$$

Puede sustituirse por

$$
K_{b i}=\frac{\alpha I_{o}}{K_{e} H}\left(1-e^{-K_{e} H}\right)
$$

La pérdida por sedimentación de los patógenos, depende de que tanto se adhieren a las partículas o que tantos quedan libres flotando. De tal manera que el total de bacterias se subdividen en flotantes y adheridas a las partículas sedimentables: $N=N_{w}+N_{p}$; donde $N_{w}$ bacterias flotantes (número. $100 \mathrm{~mL}^{-1}$ ) y $N_{p}$ bacterias adheridas (número. $100 \mathrm{~mL}^{-1}$ ). La cantidad de bacterias adheridas pueden expresarse como una concentración de masa específica, 
$r$ (número. $100 \mathrm{~mL}^{-1}$ ). Por tanto el volumen específico de las partículas; $N_{p}=10^{-4} r * m$; donde $m=$ sólidos suspendidos $\left(\mathrm{mg} . \mathrm{L}^{-1}\right)$. La tendencia de la adherencia de las bacterias a las partículas se puede representar por una tendencia lineal así $K_{d}=10^{-4} \frac{r}{N_{w}}$.

Donde: $K_{d}$ es el coeficiente de tendencia $\left(\mathrm{m}^{3} \cdot \mathrm{g}^{-1}\right)$. La tasa con la que se absorben y desabsorben las bacterias a las partículas, se asume en un equilibrio así:

$N=N_{w}+K_{d} * m * N_{w}$; quedando entonces una ecuación final. $N_{w}=F_{w} * N$; donde $F_{w}$ es la fracción de las bacterias libres flotantes. $F_{w}=\frac{1}{1+K_{d} m}$;

luego $N_{p}=F_{p} * N$; donde $F_{p}$ es la fracción de bacterias adheridas.

Luego $F_{p}=\frac{K_{d} m}{1+K_{d} m}$.

Ahora, si $V_{s}$ es la velocidad de sedimentación de las partículas $\left(\mathrm{m}^{\mathrm{d}} \mathrm{d}^{-1}\right)$, la Tasa de pérdida por sedimentación puede ser representada como:

$$
K_{b s}=F_{p} \frac{V_{s}}{H}
$$

Dando como resultado la ecuación para calcular la tasa de decaimiento total:

$$
K_{b}^{\prime}=(0,8+0,02 s) 1,07^{T-20}+\frac{\alpha I_{o}}{K_{e} H}\left(1-e^{-K_{e} H}\right)+F_{p} \frac{V_{s}}{H}
$$

En la Tabla 8 se ilustra dicho decaimiento.

Tabla 8. Tasa de decaimiento de patógenos.

\begin{tabular}{|c|c|c|c|c|}
\hline \multirow{2}{*}{ TRAMO } & $K_{b 1}$ & $K_{b i}$ & $K_{b s}$ & $K_{b}^{\prime}$ \\
\cline { 2 - 5 } & $\mathbf{d}^{\mathbf{- 1}}$ & $\mathbf{d}^{\mathbf{1}}$ & $\mathbf{d}^{\mathbf{- 1}}$ & $\mathbf{d}^{\mathbf{- 1}}$ \\
\hline Ignacio & 0,6 & 29,9 & $2,1 \mathrm{E}-05$ & 30,5 \\
\hline Judía & 0,8 & 24,6 & $1,2 \mathrm{E}-05$ & 25,4 \\
\hline Esperanza & 0,9 & 27,9 & $1,9 \mathrm{E}-05$ & 28,7 \\
\hline Botánico & 0,9 & 30,8 & $3,0 \mathrm{E}-05$ & 31,7 \\
\hline Pórtico & 1,0 & 28,0 & $2,4 \mathrm{E}-05$ & 29,1 \\
\hline Callejuelas & 1,4 & 22,4 & $1,6 \mathrm{E}-04$ & 23,8 \\
\hline Caneyes & 1,5 & 18,2 & $9,3 \mathrm{E}-05$ & 19,6 \\
\hline
\end{tabular}

\section{Estimación de la tasa de sedimentación}

La velocidad de sedimentación se calculó a partir de la ecuación de Stokes:

$$
V_{s}=\alpha \frac{g}{18}\left(\frac{\rho_{s}-\rho_{w}}{\mu}\right) d^{2}
$$

Donde: $V_{s}=$ velocidad de sedimentación $\left(\mathrm{cm} \cdot \mathrm{s}^{-1}\right) ; \alpha=$ factor reflectivo, aproximadamente 1,$0 ; \rho_{s}=$ densidad de la partícula $\left(\mathrm{g} . \mathrm{m}^{-3}\right) ; \rho_{w}=$ densidad del agua; $\mu=$ viscosidad dinámica del agua $\left(0,014\right.$ g. $\left.\mathrm{m}^{-3}\right) ; d=$ diámetro de la partícula $(\mathrm{cm})$ [15].

$$
V_{s}=0,033634 \alpha\left(\rho_{s}-\rho_{w}\right) d^{2}
$$

Donde $V_{s}\left(\mathrm{~m} . \mathrm{d}^{-1}\right) ; d(\mu \mathrm{m}) ; \rho_{s}, \rho_{w}\left(\mathrm{~g} . \mathrm{m}^{-3}\right) . \mathrm{La}$ densidad de la materia orgánica en el río tiene un valor cercano a $1,027 \mathrm{gm}^{-3}$, y el diámetro de las partículas oscilan entre 40 y $80 \mu \mathrm{m}$, permitiendo calcular la velocidad y la tasa de sedimentación al relacionarla con la profundidad de cada tramo en el río, como se ilustra en la Tabla 9.

Tabla 9. Sedimentación de la DBO en el río Frío.

\begin{tabular}{|c|c|c|c|}
\hline \multirow{2}{*}{ TRAMO } & $\boldsymbol{H}$ & $\mathbf{V s}$ & $\mathbf{K s}$ \\
\cline { 2 - 4 } & $\mathbf{m}$ & $\mathbf{m . d}^{\mathbf{- 1}}$ & $\mathbf{d}^{\mathbf{- 1}}$ \\
\hline Ignacio & 0,24 & 0,2 & 0,85 \\
\hline Judía & 0,48 & 0,2 & 0,42 \\
\hline Esperanza & 0,32 & 0,2 & 0,63 \\
\hline Botánico & 0,20 & 0,2 & 1,00 \\
\hline Pórtico & 0,25 & 0,2 & 0,80 \\
\hline Callejuelas & 0,30 & 0,2 & 0,67 \\
\hline Caneyes & 0,30 & 0,2 & 0,67 \\
\hline
\end{tabular}

\section{Remoción de la materia orgánica particulada, MOP}

En el río la remoción de la materia orgánica sucede en dos procesos simultáneamente: oxidación y sedimentación. La remoción de la DBO (L) en el río definida como:

$\frac{\partial L}{\partial t}=-U \frac{\partial L}{\partial x}-k_{r} L$, en estado estable sería:

$0=-U \frac{d L}{d x}-k_{r} L$

Se asume que en la localización de la descarga ocurre una mezcla puntual, definida como:

$$
L_{0}=\frac{Q_{w} L_{w}+Q_{r} L_{r}}{Q_{w}+Q_{r}}
$$

Donde, $Q_{r} L_{r} ;+Q_{w} L_{w}$ es la carga definida por el caudal y la concentración del río y el vertido respectivamente.

Se calcula la DBO en el tiempo t, de acuerdo con la ecuación: $L=L_{0} e^{-\frac{K_{r}}{U} x}$, luego, la DBO, aguas abajo en cualquier tramo se define como: 


$$
k_{r}=\frac{\left(\ln L_{0}-\operatorname{Ln} L\right) * U}{x}
$$

Si $K_{r=} K_{d}+K_{s}$; siendo, $K_{d}$ la tasa de desoxigenación y $K_{s}$ la tasa de sedimentación de la DBO, se calcula por la relación entre la velocidad de sedimentación de la DBO que oscila entre 0,1 y 0,5 m.d. $\mathrm{d}^{-1}$ con una media de $0,5 \mathrm{~m} \cdot \mathrm{d}^{-1} \mathrm{y}$ la profundidad, $H$, en (m), [14].

$$
k_{s}=\frac{v_{s}}{H}
$$

Para observar cuál de los tramos presenta más remoción, se debe observar la Tabla 10.

Tabla 10. Tasa de remoción (Kr) de la DBO.

\begin{tabular}{|c|c|c|c|}
\hline \multirow{2}{*}{ TRAMO } & $\mathbf{K d}$ & $\mathbf{K s}$ & $\mathbf{K r}$ \\
\cline { 2 - 4 } & $\mathbf{d}^{\mathbf{- 1}}$ & $\mathbf{d}^{\mathbf{1}}$ & $\mathbf{d}^{\mathbf{- 1}}$ \\
\hline Ignacio & 0,36 & 0,85 & 1,20 \\
\hline Judía & 0,17 & 0,42 & 0,58 \\
\hline Esperanza & 0,32 & 0,63 & 0,95 \\
\hline Botánico & 0,36 & 1,00 & 1,36 \\
\hline Pórtico & 0,32 & 0,80 & 1,12 \\
\hline Callejuelas & 0,45 & 0,67 & 1,12 \\
\hline Caneyes & 0,41 & 0,67 & 1,07 \\
\hline \multicolumn{2}{|l}{}
\end{tabular}

\section{Capacidad de autopurificación del río Frío}

La autopurificación se relaciona con la capacidad de asimilación de cargas contaminantes en fuentes hídricas de origen lótico o léntico, En nuestro caso, el río Frío es una fuente de agua potable y captación de vertidos domésticos en la zona baja. El río puede diluir hasta una determinada cantidad de carga orgánica, que contiene carbono y nitrógeno orgánico y arenas que adhieren patógenos. El modelo matemático unidimensional puede calcular el déficit de la demanda de oxígeno disuelto generada en la columna de agua y el fondo del río de acuerdo con las tasas de remoción y nitrificación, la pérdida de patógenos o decaimiento es inversamente proporcional al déficit de oxígeno en el agua.

De acuerdo con la metodología de modelos predictivos de calidad del agua calibrados y validados, propuesta por [4, 17], es posible predecir la capacidad de asimilación de carga orgánica en ríos de montaña, calculando las tasas cinéticas de oxidación de dicha materia orgánica para aplicarlas a un modelo unidimensional de calidad de agua. En esta investigación se predice la capacidad de asimilación de carga orgánica del río Frío, estimando la carga máxima que puede asimilar el río, aplicando el modelo QUAL2K versión 2,11, en el que simula el comportamiento de una determinante de calidad de concentración $c$, en un tiempo $t$ para cada tramo en el río. Para todo contaminante $\mathrm{C}$, esta ecuación puede escribirse como:

$$
\partial c / \partial t=\frac{\partial\left(A_{c} \cdot E \frac{\partial c}{\partial x}\right)}{A_{c} \partial x}-\frac{\partial\left(A_{c} U c\right)}{A_{c} \partial x}+\frac{d c}{d t}+\frac{s}{V}
$$

Donde, $A_{c}$ es el área de la sección transversal $\left(\mathrm{L}^{2}\right)$; $E$ es el coeficiente de dispersión $\left(\mathrm{L}^{2} \mathrm{~T}^{-1}\right) ; \frac{d c}{d t}$ los cambios en componentes de crecimiento y consumo $\left(\mathrm{LT}^{-1}\right) ; s$ las fuentes externas o vertidos $\left(\mathrm{MT}^{-1}\right)$, en la que $\mathrm{M}$ es la masa (M); L la distancia (L); $\mathrm{T}$ es el tiempo y $\mathrm{C}$ es la concentración $\left(\mathrm{ML}^{-3}\right)$. Como $M=V^{*} C$, se puede decir que $V x=A x^{*} d x$, que es el incremento del volumen $\left(\mathrm{L}^{3}\right)$. Si consideramos que el flujo en la corriente es estacionario $\frac{\partial Q}{\partial t}=0$, entonces $\frac{\partial V}{\partial t}=0$. El término $\frac{\partial c}{\partial t}$ es el gradiente local de concentraciones. Este último incluye el efecto de los cambios en los componentes, así como dispersión, advección, fuentes/vertidos y diluciones. Bajo condiciones de estado estacionario la derivada local es cero, es decir, la $\frac{\delta c}{\delta t}=0$, los cambios incluyen las reacciones químicas y biológicas y las interacciones que ocurren en la corriente, por ejemplo la reaireación, respiración y fotosíntesis de algas y decaimiento de coliformes. El modelo considera que el régimen hidráulico de la corriente es estado estacionario $\frac{\delta c}{\delta t}=0$, por tanto el balance hidrológico para un elemento computacional puede escribirse como: $\left(\frac{\partial Q}{\partial x}\right)_{i}=\left(Q_{x}\right)_{i}$; donde $\left(Q_{x}\right)_{i}$ es la suma de los afluentes externos y aprovechamientos para dicho elemento. Esto indica que el caudal de aprovechamiento en el canal es fundamental para realizar el balance de masa de la carga contaminante del río.

La calibración del modelo QUAL2K, versión 2.11 se realizó en una investigación preliminar, [3], aplicando el método GLUE, [17], basada en simulaciones de Monte Carlo, [18], cuya función 
objetivo, el coeficiente de determinación $R 2$, se calcula con los datos modelados y observados de oxígeno disuelto, DBO, ion amonio y fósforo soluble reactivo, entre otros parámetros.

La asimilación de la materia orgánica está en función de cada determinante, con un respectivo factor de asimilación en el agua ADZ-Quasar, [19]. El modelo considera advección, y dispersión longitudinal y reacción de primer orden. La ecuación general de balance de masa para un determinante de calidad del agua $C$ es:

$$
\frac{d C_{j}(t)}{d t}=\frac{1}{T r}\left(e^{-k \tau} \cdot C_{a}(t-\tau)-C_{j}(t)\right)-k \cdot C_{j}(t)
$$

Donde, $C_{j}$ es la concentración del determinante en la salida del tramo; $C_{a}$ es la concentración del determinante a la entrada al tramo; $k$ es la tasa de reacción, $\operatorname{Tr}$ es el tiempo de residencia, calculado como: $\operatorname{Tr}=\bar{t}-\tau$, donde $\tau$ es el tiempo de arribo, $\bar{t}$ es el tiempo medio de viaje absoluto, relacionados con la dispersión en el río. De acuerdo con [20], el factor de asimilación $a j$, permite relacionar la carga contaminante a la entrada del tramo $W_{j}$, con la concentración que resulta aguas abajo $C_{j}$ así: $C_{j}=\frac{W_{j}}{a_{j}}$. Para una condición de estado estable, la concentración aguas abajo y el factor de asimilación en cada tramo en el río está dada por las ecuaciones (34) y (35):

$$
\begin{aligned}
& c=\frac{e^{-k \tau} \cdot W}{Q \cdot(1+k \cdot(\bar{t}-\tau))} \\
& a=\frac{Q \cdot(1+k \cdot(\bar{t}-\tau))}{e^{-k \tau}}
\end{aligned}
$$

Los factores de asimilación para los diferentes determinantes son:

$$
\begin{array}{cc}
\mathrm{a}_{\mathrm{c}}=\mathrm{Q} & \text { S.Cons } \\
a_{\mathrm{L}}=\frac{\mathrm{Q} \cdot\left(1+\mathrm{k}_{\mathrm{r}} \cdot(\overline{\mathrm{t}}-\tau)\right)}{\mathrm{e}^{-\mathrm{k}_{\mathrm{r}} \cdot \tau}} & \text { DBO } \\
a_{N T K}=\frac{Q \cdot\left(1+k_{n a} \cdot(\bar{t}-\tau)\right)}{e^{-k_{n a} \cdot \tau}} & \text { NTK } \\
a_{C T}=\frac{Q \cdot\left(1+k_{b} \cdot(\bar{t}-\tau)\right)}{e^{-k_{b} \cdot \tau}} & \begin{array}{l}
\text { Coli. } \\
\text { Fecal }
\end{array}
\end{array}
$$

$$
\begin{gathered}
a_{S S T}=\frac{Q \cdot\left(1+k_{s} \cdot(\bar{t}-\tau)\right)}{e^{-k_{s} \cdot \tau}} \quad \mathrm{SST} \\
a_{O D}=\frac{Q \cdot\left(1+k_{a} \cdot(\bar{t}-\tau)\right)}{e^{-k_{a} \cdot \tau}+\varsigma \cdot(\bar{t}-\tau)} \\
\varsigma=k_{a} \cdot \frac{o_{s}}{O D_{U}} 4,57 \frac{N T K}{O D_{U}}-k_{a} \cdot \frac{D B O}{O D_{U}}
\end{gathered}
$$

Donde: $k_{r}, k_{n a}, k_{s}, k_{a}$, son las tasas de remoción, nitrificación, sedimentación y reaireación, respectivamente. $o_{s}$ es el oxígeno saturado que se calcula por la ecuación (APHA) [21].

En las Figuras 4, 5, 6, 7 y 8 se ilustra el modelamiento de la autopurificación, basada en el comportamiento del oxígeno y los contaminantes orgánicos como el carbono orgánico disuelto, definido como DBO rápida "Cf"; el amonio y los patógenos) al igual que los contaminantes inorgánicos, como es el caso del material de arrastre definido por los sólidos suspendidos.

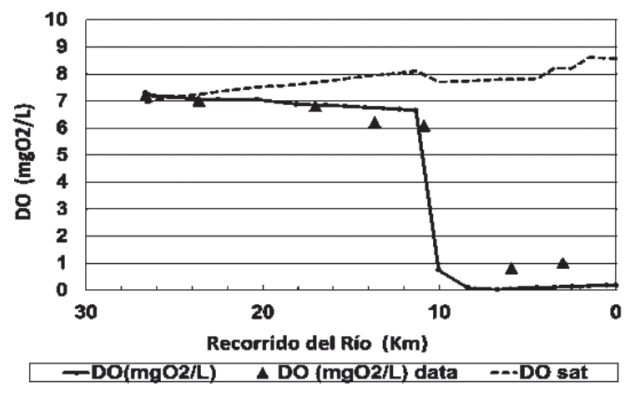

Figura 4. Comportamiento del oxígeno disuelto.

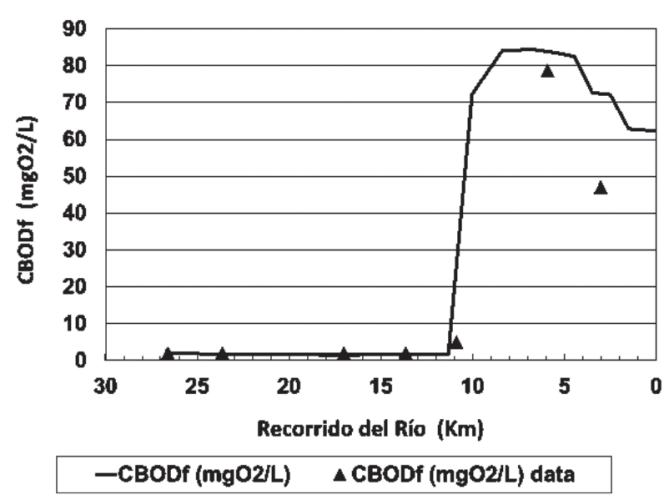

Figura 5. Modelado de la DBOC rápida en el río Frío. 


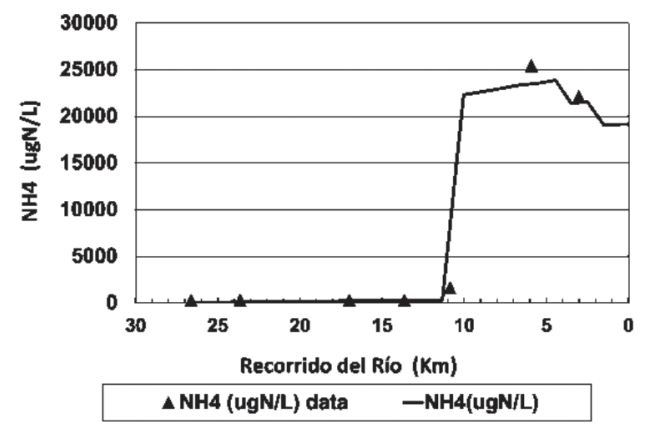

Figura 6. Modelado del amonio en el río Frío.

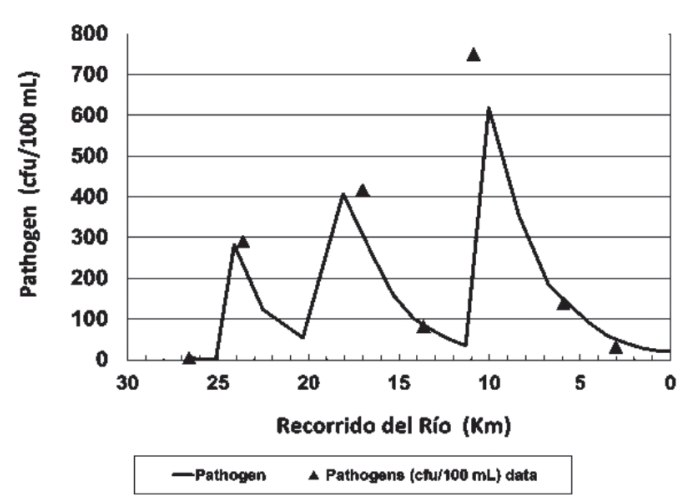

Figura 7. Modelado de los patógenos en el río Frío.

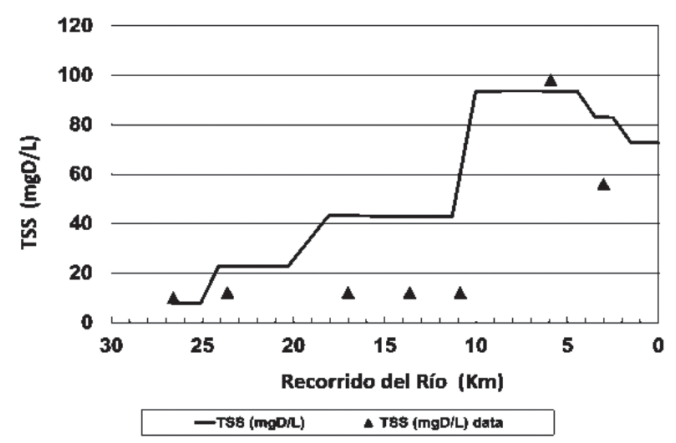

Figura 8. Modelado de los sólidos totales en el río Frío.

\section{CONCLUSIONES}

La morfometría del río Frío presenta un canal de alta pendiente (7\%) en la zona alta y baja pendiente $(2 \%)$ en la zona inferior, lo que repercutiría en una merma de velocidad durante el recorrido, pero se observa que no hay variación respecto de esta variable, manteniéndose en un promedio de 0,6 $\mathrm{ms}^{-1}$ y una sección transversal media de $9,19 \mathrm{~m}^{2}$; lo que repercute en un coeficiente de dispersión longitudinal elevado en la zona baja con un rango de 6 a $16 \mathrm{~m}^{2} \mathrm{~s}^{-1}$, esto debido a una elevada rugosidad de 0,15 , debido a la presencia de roca en el fondo del río durante todo el recorrido.

El segmento hidráulico modelado presenta una longitud de 26,6 km, con 11,7 horas de viaje, el fotoperiodo que se presenta en la zona es de 12,53 horas, para mayo de 2012. La temperatura del aire oscila entre 18 y $28^{\circ} \mathrm{C}$ y la del agua mantiene una diferencia de $5^{\circ} \mathrm{C}$. El río cae desde un piso térmico de páramo bajo de $2091 \mathrm{msnm}$ a una zona cálida de $715 \mathrm{msnm}$, observándose una elevación de la conductividad en la zona baja con una oscilación de 100 a $650 \mu \mathrm{S} . \mathrm{cm}^{-1}$, lo que permite comprobar el aumento de la contaminación, al igual que el aumento de la alcalinidad por la presencia de amonio, oscilando entre 26,6 a $170 \mathrm{mg} \cdot \mathrm{CaCO}_{3} \mathrm{~L}^{-1}$; mientras que el $\mathrm{pH}$ no sufre alteraciones ( 7 a 8 ).

Un río de montaña suele tener suficiente reaireación $\left(>50 \mathrm{~d}^{-1}\right)$ dependiendo de una buena profundidad (> $1,5 \mathrm{~m})$, alta velocidad $\left(>0,5 \mathrm{~m}, \mathrm{~s}^{-1}\right)$ y baja radiación solar. Las ecuaciones empíricas tienen en cuenta solo la profundidad y la velocidad y esto las hace muy específicas para ciertos cuerpos lóticos. En ríos de baja profundidad $(<1 \mathrm{~m})$ la reaireación se ve afectada porque no se tiene la suficiente interface de agua para hacer una absorción alta de oxígeno atmosférico, al mismo tiempo las partículas de arena aceleran la desoxigenación y la alta rugosidad aumenta la reaireación. Se encontró que un río de montaña de baja profundidad puede presentar tasas de reaireación entre 0,8 y $6,4 \mathrm{~d}^{-1}$, de acuerdo con la validación del oxígeno en el río Frío, con un límite de confianza del $95 \%$.

La tasa de reaireación evaluada experimentalmente para el río Frío, usando las concentraciones de oxígeno disuelto y oxígeno saturado validados en campo, arrojó una tasa media de $\left(\mathrm{k}_{\mathrm{a}}=3,24 \mathrm{~d}^{-1}\right)$, presentando variaciones de $6,4 \mathrm{~d}^{-1}$, en el tramo Ignacio a $1 \mathrm{~d}^{-1}$, en promedio para los tramos: Judía, Esperanza, Botánico y Pórtico; ya en los dos últimos tramos la tasa aumenta a 2 y $3 \mathrm{~d}^{-1}$ debido al aumento del caudal, la profundidad y la velocidad. Si comparamos la tasa experimental con la calculada por las ecuaciones empíricas, como: Texas $\left(5,37 \mathrm{~d}^{-1}\right)$, O'Connor $\left(0,08 \mathrm{~d}^{-1}\right)$, Churchill $\left(29,74 \mathrm{~d}^{-1}\right)$ y Owens $\left(45,04 \mathrm{~d}^{-1}\right)$, se observa la más ajustada a la experimental es la de Texas, con un 
( $\mathrm{T}$ crítico $=1,80)$ y un $(\mathrm{T}$ calculado $=0,002)$ para un $95 \%$ de confianza y 11 grados de libertad.

La tasa de desoxigenación media obtenida por el método experimental $\left(\mathrm{k}_{\mathrm{d}}=0,38 \mathrm{~d}^{-1}\right)$ y por el método Bosko $\left(\mathrm{k}_{\mathrm{d}}=0,34 \mathrm{~d}^{-1}\right)$ no presentaron diferencias significativas $(\mathrm{T}$ critico $=1,83, \mathrm{~T}$ calculado $=0,32)$ con una cola y una confianza del $95 \%$. Lo que quiere decir que pueden usarse las dos ecuaciones para dicha medición con (9) grados de libertad. La medición realizada con la ecuación Hydroscience refleja un tasa media de $\left(\mathrm{k}_{\mathrm{d}}=2,14 \mathrm{~d}^{-1}\right)$, lo que representa un valor muy elevado respecto del rango establecido de $0,30,6 \mathrm{~d}^{-1}$. Las obtenidas en campo por el método de conversión de COT, presentan valores de $0,5 \mathrm{~d}^{-1}$, en promedio para los tramos iniciales: Ignacio, Judía y Esperanza, mientras que aguas abajo (Botánico) se presenta un descenso a $0,4 \mathrm{~d}^{-1}$ y $0,2 \mathrm{~d}^{-1}$ debido al aumento de la carga de carbono orgánico. Es evidente que se debe optar más, por la aplicación de un método experimental que el de botella, ya que no representa las condiciones hidrogeométricas del río.

El cálculo de la tasa de nitrificación desarrollado por el método de conversión de DBON a partir del NTK presenta una media de $\left(\mathrm{k}_{\mathrm{n}}=11,52 \mathrm{~d}^{-1}\right)$, ( $\mathrm{T}$ crítico $=1,08, \mathrm{~T}$ calculado $=1,94)$, existiendo una diferencia significativa que logra superar la referencia de $1,2 \mathrm{~d}^{-1}$ propuesta para ríos en Colombia. El comportamiento de la concentración de NTK, en los diferentes tramos del río Frío mantiene una actividad baja en los cuatro primeros tramos, lo que cambia al aumentar dicha concentración para los tramos Pórtico, Callejuelas y Caneyes, donde el amonio y el nitrógeno orgánico se oxidan a nitrato a una velocidad de 2,3\% por hora, dejando nitrógeno sin oxidar debido a la alta desoxigenación (3 veces más alta).

La presencia de patógenos en fuentes naturales cercanas a asentamientos urbanos es evidente, debido a la alta concentración de heces fecales por presencia de vertidos domésticos (600 ufc. 100 $\mathrm{mL}^{-1}$ ). En un río de montaña de poca profundidad el decaimiento de los patógenos se presentan con una tasa de mortalidad natural de $1,3 \mathrm{~d}^{-1}$, es decir, que solo duran 18,5 horas vivos. Por otra parte, se genera también muerte por la alta radiación solar, la que presenta una tasa de $26,3 \mathrm{~d}^{-1}$ debido a la ubicación geográfica en la zona ecuatorial donde la radiación llega a $36,7 \mathrm{cal} \mathrm{cm}^{-2} \mathrm{~d}^{-1}$. La pérdida de patógenos por sedimentación es muy baja (1E-4 $\left.\mathrm{d}^{-1}\right)$, luego no afecta a la sumatoria de la tasa de decaimiento que se encuentra entre 23 y $30 \mathrm{~d}^{-1}$, es decir, que el decaimiento es de 1,5 a $2,1 \%$ cada minuto. Esto indica una vulnerabilidad muy alta de los patógenos, convirtiéndose en un problema de aporte al fondo del río como detritos o la generación de enfermedades, en su etapa vital. El comportamiento de la tasa en los tramos del río se define como homogénea en $30 \mathrm{~d}^{-1}$, para los tramos (Ignacio, Judía, Esperanza, Botánico y Pórtico), aguas abajo, presenta una disminución a $20 \mathrm{~d}^{-1}$, esto debido a la mortalidad de patógenos debido al medio anóxico y transporte de material de arrastre.

El río Frío presenta concentraciones de sólidos suspendidos que oscilan entre 10 y $98 \mathrm{mg} \mathrm{L}^{-1}$. El aumento se debe a la presencia de los vertidos domésticos, ya que estas aguas provienen de alcantarillados mixtos (doméstico-pluvial). Parte de esos SST son los sólidos suspendidos orgánicos, lo que representa la materia orgánica particulada, aproximadamente el $50 \%$ de los suspendidos totales, esto nos indica que existe igual cantidad de bacterias que arena en el río. La velocidad de sedimentación de las arenas es de $0,2 \mathrm{~m} \mathrm{~d}^{-1}$, lo que implica una acumulación alta de material particulado en el fondo del río, ocasionando disminución de la velocidad y una mayor demanda bentónica. La remoción de la DBO carbonacea media es de $\left(\mathrm{k}_{\mathrm{s}}=0,72 \mathrm{~d}^{-1}\right)$, con una tasa de sedimentación de la DBO en $\left(\mathrm{k}_{\mathrm{r}}=1,06 \mathrm{~d}^{-1}\right)$, esto indica que la DBOC se sedimenta en $70 \%$, aproximadamente.

La carga de materia orgánica que se vierte al río Frío es de 26,2 ton $\mathrm{d}^{-1}$ y la asimilación es tan solo del $10 \%$ de la carga es decir 2,6 ton $\mathrm{d}^{-1}$, en términos de DBO, sería 12,2 $\mathrm{mg} \mathrm{L}^{-1}$, manteniendo los mismos caudales de los vertidos, canal y fuente de dilución. Lo que afecta la calidad del agua a este tipo de río de montaña es la cantidad de vertidos, las altas concentraciones de estos y la cercanía entre ellos con respecto al tiempo de recorrido del río.

\section{AGRADECIMIENTOS}

El autor agradece al apoyo generado por el Convenio interinstitucional entre las UTS y la CDMB, al Dr. Luis Alfonso Camacho (Investigador de la Universidad de los Andes). 


\section{REFERENCIAS}

[1] DANE. "Estudios Postcensales 7, Proyecciones nacionales y departamentales de población 2005-2020”. Bogotá. 2010.

[2] CDMB. "Informe anual de la Red de Monitoreo de Calidad del Agua". 2012. URL: http://caracoli.cdmb.gov.co/ cai/rhc/ repmdc.html. Fecha de consulta: 18 Marzo 2015.

[3] J. Rivera. "Evaluación de la materia orgánica en el río Frío soportada en el QUAL2k versión 2.07”. Revista DYNA. Vol. 78 No 169, pp. 131-139. Octubre 2011.

[4] L. Camacho, E.A. Rodríguez, R. Gélvez, R. González, M. Medina y J. Torrres. "Caracterización De La Capacidad De Autopurificación De Ríos De Montaña”. 17 Agosto 2007. URL: http://www.docentes. unal.edu.co/ricagonzalezp/docs/VF-ArticuloICongAgAmb_AutopurificacionRM.pdf. Fecha de consulta: 10 Octubre 2012.

[5] OMM. "Guía de Prácticas Hidrológicas -168". 1994. URL: www.inamhi.gov.ec/educativa/ WMOSPA.pdf. Fecha de consulta: 18 Marzo 2009.

[6] ICONTEC. "Guía para el Muestreo de Aguas de Ríos y Corrientes NTC-ISO 5667-6". Bogotá. 2006.

[7] Hydroscience, Inc. "Simplified Matematical Modeling of Water Quality". Washington D.C: Mitre Corporation and USEPA, Water Programs. 1971.

[8] D.J. y. D. O'Connor. "Mechanism of Reaeration in Natural Streams". Trans. Am. Soc. Civil Engin. 123, pp. 641-666, 1958.

[9] M.A. Churchill, H.L. Elmore and R.A. Buckingham. "Prediction of Streams Reaereation Rates". Journal of the Sanitary Engineering Division. Vol. 88 No 4, pp. 1-46. ASCE. 1962.

[10] M. Owens, RW Edwards and JW. Gibbs. "Some Rearation Studies in Streams". Int. J. Air Water Poll. 8, pp. 469-486. 1964.

[11] EC Tsivoglou, JR Wallace. "Characterizaction of Stream Reaeration Capacity". USEPA, Report N ${ }^{\circ}$ EPA - R3-72-012. 1972.
[12] Texas Water Development Board. "Simulation of Water Quality in Stream and Canals, Program Documentation and User's Manual". Austin, TX, 1970.

[13] A. F. Gaudy and E. T. Gaudy. "Microbiology for Enviromental Scientists and Engineers". New York. Mc Graw Hill. 1980.

[14] S. CHAPRA. "Surface Water Quality Modelling”. New York: Mc Graw Hill. 1997.

[15] R. Thomann and J. Mueller. "Principles of Surface Water Quality Modeling and Control". New York, USA. Harper \& Row. 1987.

[16] D. Di Toro, J. Fitzpatrik y R. Thoman. "Programa de Calidad del Agua Análisis Simulación (WASP) y el Programa de Verificación de modelo (MVP)". USEPA. Duluth, MN, Nueva Jersey. 1981.

[17] Beven J. "Generalized Likelihood Uncertainty Estimation (GLUE) - User manual”. 1998.

[18] Lees and Wagener. "Monte-Carlo Analysis Toolbox (MCAT)" 2000. URL: http://www.researchgate.net/profile/ Thorsten_Wagener/publication/267818673_ Monte-Carlo_Analysis_Toolbox_User_ Manual/links/5491a1120cf23b7c974c1281. pdf

[19] M. Lees, L. Camacho and P. Whitehead, "Extension of the QUASAR water quality model to incorporate dead-zone mixing". 1998.

[20] L.A. Camacho. "Calibración y análisis de la capacidad predictiva de modelos de transporte de solutos en un río de montaña colombiano". Revista Avances en recursos hidráulicos. Vol. 14. 2006.

[21] Steve Chapra, Greg Pelletier and Hua Tao. "QUAL2K Version 2.11b8 A Modeling Framework for Simulating River and Stream Water Quality”. Tufts University, Medford, MA, 2008.

[22] Corporación autónoma regional para defensa de la meseta de bucaramanga (CDMB). "Repositorio de Archivos de Interés e Informes Periódicos de la Red de Monitoreo de Corrientes". CDMB. Bucaramanga. Informe Anual y Semestral de Calidad. 2012. 